\title{
EFFECT OF FIELD OF VIEW ON THE ACCURACY OF CONE BEAM COMPUTED TOMOGRAPHIC ASSESSMENT OF ALVEOLAR BONE LOSS IN PERIODONTAL DEFECTS
}

\author{
Enas Anter*, Mohammed Khalifa Zayet** and Sahar Hosny El-Dessouky***
}

\begin{abstract}
Objectives: This study aimed to determine the effect of changing the field of view on the accuracy of Cone Beam Computed Tomographic assessment of alveolar bone loss in periodontal defects.

Materials and methods: 73 natural human teeth naturally attached to ten dry human jaws were used with small linear gutta percha pieces with central indentation glued on their facial and lingual/ palatal surfaces at the place of CEJ, to be used as marker for alveolar bone level measurements both directly using digital caliber with an accuracy of $0.01 \mathrm{~mm}$ and radiographically on three CBCT images for each tooth obtained at three different field of views (FOV) $(80 \times 80 \mathrm{~mm}, 100 \times 100 \mathrm{~mm}$ and $200 \times 100 \mathrm{~mm}$ ) using a standardized voxel size of $0.2 \mathrm{~mm}$, the direct and CBCT measurements were then compared.
\end{abstract}

Results: there was no statistically significant difference between the mean CBCT measurements errors at different FOVs. The average CBCT measurements error in the three FOVs $(80 \times$ $80 \mathrm{~mm}, 100 \times 100 \mathrm{~mm}$ and $200 \times 100 \mathrm{~mm}$ ) were $0.23 \pm 0.09 \mathrm{~mm}, 0.24 \pm 0.10 \mathrm{~mm}$ and $0.21 \pm 0.09 \mathrm{~mm}$ respectively.

Conclusions: The FOV size has no significant effect on the CBCT measurements accuracy of the alveolar bone level.

KEY WORDS: CBCT, Field of view, Alveolar bone loss.

\section{INTRODUCTION}

Alveolar bone loss in periodontal defects is a common dental problem and accurate assessment of the true extension of the periodontal defect is essential for proper formulation of a suitable treatment plan. Besides, prior to formulating any dental treatment plan, a thorough examination of the supporting periodontium is required since accurate determination of alveolar bone height and architecture around the dentation is very important to obtain ideal functional and esthetic prosthetic reconstruction ${ }^{1-3}$.

* Assistant Lecturers of Oral and Maxillofacial Radiology, Faculty of Oral and Dental Medicine, Cairo University ** Associate Professor of Oral and Maxillofacial Radiology, Faculty of Oral and Dental Medicine, Cairo University 
In periodontal defects assessment, the current radiographic approaches, including intraoral and panoramic radiography, have shown several limitations in their reliability mainly owing to their $2 \mathrm{D}$ nature. The solution of such a problem was to shift to $3 \mathrm{D}$ diagnostic imaging of the jaws, and with the evolution of CBCT it offered a cheaper, faster, small sized and dose sparing alternative to $\mathrm{CT}$ with higher spatial resolution and isotropic voxels ${ }^{4-6}$.

One of the great advantages of CBCT is that it provides the possibility of adjusting the size of the FOV according to the task for which the scan is made ${ }^{6,7}$. FOV is determined by detector size and shape, beam projection geometry and beam collimation, which limits radiation exposure to a particular region of interest.

Increased FOV is usually associated with increased scattered radiation, resulting in more noise and decreased SNR which is reflected on the image contrast. Yet the reduction of the FOV size could result in truncation effect artifact with the peripheral areas of the scan appearing to be less dense (darker) and contain more noise $\mathrm{e}^{8-10}$.

Does FOV size alone affect accuracy of CBCT linear measurements or not? No study -to best of our knowledge- was found discussing this point in valid methodological settings.

For that our study was made to evaluate the accuracy of CBCT in assessment of alveolar bone loss in periodontal defects, and to investigate the effect of FOV size on this accuracy.

\section{MATERIALS AND METHODS:}

The study was conducted on 73 natural human teeth including 30molars (12 lower and 18 upper), 19 premolars (seven lower and 12 upper) and 24 anterior teeth (12 lower and 12 upper), they were naturally attached to ten dry human jaws (five maxillae and five mandibles). Before conducting the study an ethical approval on the study design was obtained from the Ethics Committee for research of the Faculty of oral and dental medicine, Cairo University.

The sample size was previously calculated based on a pilot study made on 11 teeth and it was found to be 12 teeth, and to account for any drop out a sample size of 15 teeth was recommended.

On sample preparation Small pieces of guttapercha 'Gp' cones were glued on the teeth at the place of CEJ both facially and lingualy or palataly to act as standardized fiducial references compensating for the faded CEJ due to dehydration of the teeth as adopted in five previous studies ${ }^{11-15}$, then Soft tissue simulation was made by adapting eight sheets of softened pink on the skull and mandible to cover them providing approximately $12 \mathrm{~mm}$ thickness of pink wax which is consistent with what was recommended by Caldas et al $\mathbf{2 0 1 0}^{\mathbf{1 6}}$

Then the samples were scanned using a Promax ${ }^{\circledR}$ 3DMid CBCT device (Planmeca Oy, Helsinki, Finland). Each skull and mandible assembly was scanned three times using the same voxel size but at three different FOVs in three different protocols as follows:

- Protocol (1): FOV of $80 \mathrm{~mm}$ height and 80 mm width, voxel size $200 \mu$, image matrix size of $401 \times 401,90 \mathrm{kV}$ and $10 \mathrm{~mA}$ using pulsed scanning time of $12.348 \mathrm{sec}$. and the DAP "Dose area product” was $1092.8 \mathrm{mGy} \mathrm{cm} 2$. (Figure 1)

- Protocol (2): FOV of $100 \mathrm{~mm}$ height and $100 \mathrm{~mm}$ width, voxel size $200 \mu$, image matrix size of $501 \times 501,90 \mathrm{kV}$ and $10 \mathrm{~mA}$ using pulsed scanning time of $12.345 \mathrm{sec}$. and the DAP was $1092.8 \mathrm{mGy} \mathrm{cm}{ }^{2}$. (Figure 2)

- Protocol (3): FOV of $100 \mathrm{~mm}$ height and 200 mm width, voxel size $200 \mu$, image matrix size of $1001 \times 1001,90 \mathrm{kV}$ and $10 \mathrm{~mA}$ using pulsed scanning time of $18 \mathrm{sec}$. and the DAP was $1555.9 \mathrm{mGy} \mathrm{cm}^{2}$. (Figure 3) 
A single well trained observe made all the CBCT measurements after one week of training on the software used in this study (Planmeca Romexis viewer 3.5.1.R/). Alveolar bone loss was measured as the distance from the gutta-percha at the CEJ to the alveolar crest $\mathrm{AC}$, at six different positions in each tooth which were:

- Mesio-buccal 'MB': at the mesial end of the GP piece on the buccal aspect of the tooth.

- Disto-buccal 'DB': at the distal end of the GP piece on the buccal aspect of the tooth.

- Buccal 'B': at the central indention of the GP piece on the buccal aspect of the tooth.

- Mesio-lingual 'ML': at the mesial end of the GP piece on the lingual aspect of the tooth.

- Disto-lingual 'DL': at the distal end of the GP piece on the lingual aspect of the tooth.

- Lingual 'L': at the central indention of the GP piece on the buccal aspect of the tooth.

The gold standard measurements were taken directly on the dry jaws using an electronic digital caliber IOS-USA ${ }^{\circledR}$ [Yiwu Windex Import \& Export Co., Ltd] with an accuracy of $0.01 \mathrm{~mm}$.
For each position, the measurements were taken three times and their average was considered as the gold standard.

The CBCT measurements were taken using "Planmeca Romexis viewer 3.5.1.R” software with the distance measurement tool. The measurements were taken on corrected sagittal image slices for the teeth (Figures 1, $2 \& 3$ ). All the CBCT measurements were taken three times at three different sessions and the average of the three measurements was considered the final one.

Finally all the measurements including the gold standard and CBCT measurements of the different scans were tabulated, compared, and statistically analyzed.

Estimation of the measurements error between direct (gold standard) measurements (A) and CBCT measurements (B) was assessed according to the following equation: Absolute measurement error $(\mathrm{AME})=[\mathrm{B}-\mathrm{A}]$.

Friedman's test was used to compare between the average AME in different FOVs for all the teeth collectively, in different teeth types (anteriors, premolars and molars) separately, and on facial and lingual teeth surfaces.

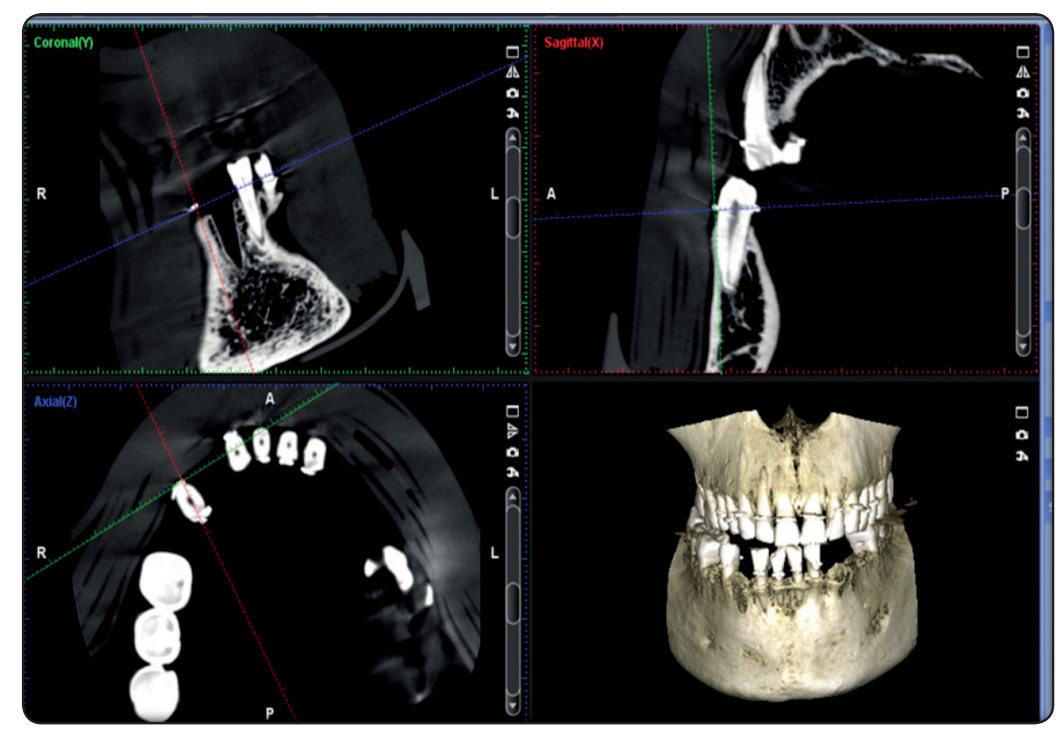

Fig. (1) The explorer screen of Romexis viewer with the $\mathrm{x}, \mathrm{y}$ and $\mathrm{z}$ planes adjusted to provide a corrected cross sectional image of the tooth aligned with its long axis on the sagittal view. This scan is of protocol (1) "FOV is $80 \times 80 \mathrm{~mm}$ and voxel size is $200 \mu$ ". Note the orientation lines positions for measurements standardization 

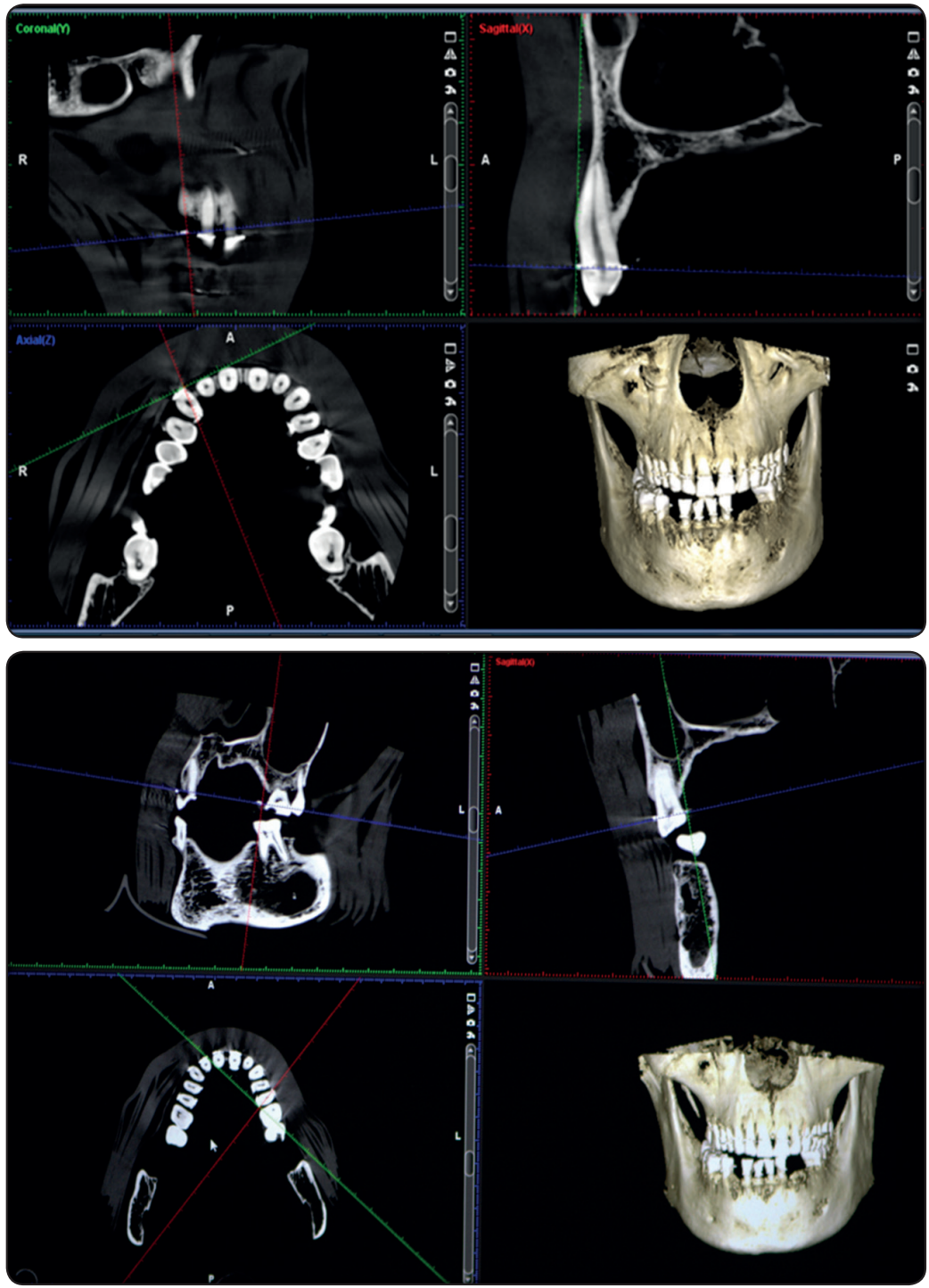

Fig. (2) The explorer screen of Romexis viewer with the $\mathrm{x}, \mathrm{y}$ and $\mathrm{z}$ planes adjusted to provide a corrected cross sectional image of the tooth aligned with its long axis on the sagittal view. This scan is of protocol (2) "FOV is $100 \times 100 \mathrm{~mm}$ and voxel size is $200 \mu$ " Note the orientation lines positions for measurements standardization.
Fig. (3) The explorer screen of Romexis viewer with the $\mathrm{x}, \mathrm{y}$ and $\mathrm{z}$ planes adjusted to provide a corrected cross sectional image of the tooth aligned with its long axis on the sagittal view. This scan is of protocol (3) "FOV is $200 \times 100 \mathrm{~mm}$ and voxel size is $200 \mu$ " Note the orientation lines positions for measurements standardization
Agreement between the Gold Standard and different CBCT measurements was measured using Cronbach's alpha reliability coefficient and Intra-Class Correlation Coefficient (ICC). The significance level was set at $\mathrm{P} \leq 0.05$. Statistical analysis was performed with IBM $^{\circledast}$ (IBM Corporation, NY, USA), SPSS ${ }^{\circledR}$ (SPSS, Inc., an IBM Company) Statistics Version 20 for Windows.

\section{RESULTS}

\section{Agreement (Reliability analysis):}

In assessment of the agreement between the GS and CBCT measurements at different FOVs, there was a very good agreement between them (Table 1)
TABLE (1) Results of Cronbach's alpha and ICC coefficients for agreement between Gold Standard and CBCT measurements at different FOVs.

\begin{tabular}{ccc}
\hline FOV & Cronbach's alpha & ICC \\
\hline $\mathbf{8 0} \times \mathbf{8 0}$ & 0.995 & 0.991 \\
$\mathbf{1 0 0 \times \mathbf { 1 0 0 }}$ & 0.995 & 0.990 \\
$\mathbf{2 0 0 \times \mathbf { 1 0 0 }}$ & 0.995 & 0.989 \\
\hline
\end{tabular}


Comparison between measurement errors at the different Fields of View regarding all teeth

Using Friedman's test for the comparison between measurement errors at the different FOVs regarding all teeth showed no statistically significant difference between the three Fields of View (Table 2).

\section{Comparison between different Fields of View in anterior, premolar and molar teeth}

Using Friedman's test for comparison between measurement error of different Fields of View with anterior, premolar and molar teeth showed no statistically significant difference between the three Fields of View at all surfaces (Table 3).

\section{Comparison between different Fields of View on facial and lingual teeth surfaces}

Using Friedman's test for the comparison between measurement error of different Fields of View at the buccal and lingual surfaces showed no statistically significant difference between measurement errors of the three Fields of View.

TABLE (2) Mean, standard deviation (SD) values and results of Friedman's test for the comparison between CBCT measurement errors at different FOVs regarding all the teeth included in the study

\begin{tabular}{|c|c|c|c|c|c|c|c|}
\hline \multirow{2}{*}{ Surface } & \multicolumn{2}{|c|}{$80 \times 80$} & \multicolumn{2}{|c|}{$100 \times 100$} & \multicolumn{2}{|c|}{$200 \times 100$} & \multirow{2}{*}{$P$-value } \\
\hline & Mean & SD & Mean & SD & Mean & SD & \\
\hline MB & 0.23 & 0.19 & 0.22 & 0.17 & 0.21 & 0.16 & 0.974 \\
\hline DB & 0.21 & 0.18 & 0.25 & 0.19 & 0.22 & 0.18 & 0.467 \\
\hline B & 0.22 & 0.19 & 0.25 & 0.21 & 0.19 & 0.14 & 0.052 \\
\hline ML & 0.22 & 0.22 & 0.21 & 0.23 & 0.20 & 0.18 & 0.825 \\
\hline DL & 0.26 & 0.27 & 0.28 & 0.27 & 0.21 & 0.20 & 0.148 \\
\hline $\mathbf{L}$ & 0.24 & 0.20 & 0.23 & 0.19 & 0.21 & 0.19 & 0.877 \\
\hline Overall & 0.23 & 0.09 & 0.24 & 0.10 & 0.21 & 0.09 & 0.669 \\
\hline
\end{tabular}

*: Significant at $P \leq 0.05$

TABLE (3) Mean, standard deviation (SD) values and results of Friedman's test for the comparison between measurement error of different Fields of View with anterior, premolar and molar teeth

\begin{tabular}{|c|c|c|c|c|c|c|c|c|}
\hline \multirow{2}{*}{ Teeth } & \multirow{2}{*}{ Surface } & \multicolumn{2}{|c|}{$80 \times 80$} & \multicolumn{2}{|c|}{$100 \times 100$} & \multicolumn{2}{|c|}{$200 \times 100$} & \multirow{2}{*}{$P$-value } \\
\hline & & Mean & SD & Mean & $\mathrm{SD}$ & Mean & SD & \\
\hline \multirow{7}{*}{ Anterior } & MB & 0.25 & 0.18 & 0.26 & 0.21 & 0.20 & 0.18 & 0.834 \\
\hline & DB & 0.21 & 0.14 & 0.30 & 0.21 & 0.28 & 0.23 & 0.558 \\
\hline & B & 0.22 & 0.13 & 0.23 & 0.19 & 0.23 & 0.16 & 0.956 \\
\hline & ML & 0.19 & 0.16 & 0.18 & 0.18 & 0.18 & 0.17 & 0.774 \\
\hline & DL & 0.23 & 0.24 & 0.34 & 0.38 & 0.23 & 0.25 & 0.485 \\
\hline & $\mathbf{L}$ & 0.25 & 0.23 & 0.23 & 0.23 & 0.22 & 0.25 & 0.989 \\
\hline & Overall & 0.23 & 0.09 & 0.26 & 0.11 & 0.23 & 0.10 & 0.747 \\
\hline
\end{tabular}




\begin{tabular}{|c|c|c|c|c|c|c|c|c|}
\hline \multirow{7}{*}{ Premolar } & MB & 0.18 & 0.15 & 0.17 & 0.13 & 0.21 & 0.17 & 0.502 \\
\hline & DB & 0.18 & 0.14 & 0.23 & 0.16 & 0.22 & 0.17 & 0.741 \\
\hline & B & 0.16 & 0.13 & 0.23 & 0.14 & 0.13 & 0.10 & 0.154 \\
\hline & ML & 0.22 & 0.26 & 0.24 & 0.30 & 0.24 & 0.25 & 0.984 \\
\hline & DL & 0.24 & 0.22 & 0.16 & 0.09 & 0.14 & 0.13 & 0.345 \\
\hline & $\mathbf{L}$ & 0.26 & 0.14 & 0.22 & 0.14 & 0.20 & 0.15 & 0.311 \\
\hline & Overall & 0.21 & 0.10 & 0.21 & 0.09 & 0.20 & 0.09 & 0.810 \\
\hline \multirow{7}{*}{ Molar } & MB & 0.25 & 0.22 & 0.22 & 0.15 & 0.21 & 0.15 & 0.962 \\
\hline & DB & 0.23 & 0.23 & 0.22 & 0.18 & 0.17 & 0.10 & 0.672 \\
\hline & B & 0.27 & 0.25 & 0.28 & 0.25 & 0.19 & 0.14 & 0.051 \\
\hline & ML & 0.24 & 0.24 & 0.22 & 0.23 & 0.18 & 0.12 & 0.965 \\
\hline & DL & 0.30 & 0.31 & 0.30 & 0.23 & 0.25 & 0.20 & 0.587 \\
\hline & $\mathbf{L}$ & 0.22 & 0.20 & 0.25 & 0.18 & 0.20 & 0.16 & 0.421 \\
\hline & Overall & 0.25 & 0.09 & 0.25 & 0.09 & 0.20 & 0.08 & 0.125 \\
\hline
\end{tabular}

*: Significant at $P \leq 0.05$

TABLE (4) Mean, standard deviation (SD) values and results of Friedman's test for the comparison between measurement error of different Fields of View at the buccal and lingual surfaces

\begin{tabular}{|c|c|c|c|c|c|c|c|}
\hline \multirow{2}{*}{ Surface } & \multicolumn{2}{|c|}{$80 \times 80$} & \multicolumn{2}{c|}{$100 \times 100$} & \multicolumn{2}{c|}{ 200×200 } & \multirow{2}{*}{ P-value } \\
\cline { 2 - 8 } & Mean & SD & Mean & SD & Mean & SD \\
\hline Buccal & 0.26 & 0.17 & 0.27 & 0.17 & 0.17 & 0.05 & 0.758 \\
\hline Lingual & 0.27 & 0.14 & 0.23 & 0.11 & 0.21 & 0.13 & 0.717 \\
\hline
\end{tabular}

*: Significant at $P \leq 0.05$

\section{DISCUSSION}

The application of CBCT in assessment of periodontal problems became evident in the last ten years although the research in this field is still limited.Assessment of the current literature indicates that only around $3 \%$ of the articles published on dental applications of CBCT have dealt with its applications in periodontology. ${ }^{17,18}$

On reviewing the current literature for studies discussing the quantitative accuracy of CBCT in assessment of alveolar bone level in periodontal defects, most of the studies found were comparing the CBCT accuracy to that of other imaging modalities ${ }^{19-22}$. However none of these studies was found discussing the role of acquisition settings variables such as the FOV size on the reported CBCT accuracy of measurements of periodontal defects.

However, on searching the literature for other studies discussing the effect of FOV size on CBCT linear measurements accuracy in other dental fields 
to serve as a guide line for our study, we found that no single study, to the date of writing this article, was found evaluating the effect of FOV solely on CBCT measurements accuracy. As two previous studies by Al-Rawi et al 2010 and Kamburoğlu et al 2014 were found addressing the effect of FOV on linear measurements accuracy ${ }^{23,24}$. However, up on revising their methodology we found that both FOV and voxel size were simultaneously changed in the scanning protocols. Moreover, a study by Cook et al 2015 examined the effect of CBCT acquisition variables on the CBCT measurements accuracy, and they simultaneously changed the voxel size, FOV and scan arc in the different protocols they used ${ }^{25}$. In these three studies we could not relate the reported effect on the accuracy of CBCT measurements to either of the examined variables separately.

However regarding the effect of FOV size on CBCT measurements accuracy, the results of this study revealed that there was no statistically significant difference between the mean CBCT measurement errors at different FOVs that we used in our study, this was applied on the whole data collectively and all its sub-divisions. The mean CBCT measurements errors for the small, medium and large FOVs we used were $0.23 \pm 0.09 \mathrm{~mm}, 0.24 \pm$ $0.10 \mathrm{~mm}$ and $0.21 \pm 0.09 \mathrm{~mm}$ respectively. The ICC test was used for assessment of the agreement between the CBCT measurements and the GS measurements at the three studied FOVs $(80 \times 80$ $\mathrm{mm}, 100 \times 100 \mathrm{~mm}$ and $200 \times 100 \mathrm{~mm}$ ), and it showed a very good agreement between CBCT and GS measurements in all the FOVs. The ICC values for them were $0.991,0.990$ and 0.989 respectively.

Although our hypothesis at the beginning of the study was that a significant error in CBCT measurements might be encountered at the periphery of the scan with the smallest FOV (mainly in the molars area) due to the effect of truncation cutoff artifact" (cone beam effect artifact), but this hypothesis was refuted by the lack of significance in CBCT measurements error in any site or at any FOV, as our results showed that the three used FOVs provided very close accuracy rate in all the teeth types (anterior, premolar and molar teeth) and on both facial and lingual or palatal surfaces.

We also aimed to test the validity of the hypothesis that the largest FOV should be associated with the greater measurements error. This assumption was based on the fact that the larger FOVs are usually associated with higher noise due to the increased scattered radiation, which will inversely affect the quality of the image and hence the accuracy of measurements ${ }^{7}$. However, in our work this was not possible, as the CBCT machine that we have used was automatically modifying the exposure time to obtain constant mAs programmed within the machine which was compensating for the noise associated with the increased FOV.

We also need to clarify that comparing the DAP in the CBCT protocols with different FOVs showed that both the small and medium sized FOV provide the same DAP, while the largest FOV provides DAP that is approximately 1.5 times like that of the other two protocols.

\section{CONCLUSIONS}

With the great benefit offered by CBCT regarding variable FOV options, and once the difference in accuracy between the protocols with different FOVs was not significant on either teeth types or surfaces, the operator should stick to the one which produces less patients hazard, especially if it also could improve the accuracy, as in clinical situationswith absence of the idealized environment present in the in-vitro study- metallic restorations may be encountered inside the patient's mouth and this necessitates the restriction of the FOV to avoid artifacts from these restorations. 


\section{REFERENCES}

1. Armitage G C. The complete periodontal examination. Periodontol 2000. 2004; 34: 22-33.

2. Braun X, Ritter L, Jervøe-Storm P.M, and Frentzen M. Diagnostic accuracy of CBCT for periodontal lesions. Clinical Oral Investigations. 2014; 18: 1229-36.

3. Schropp L, Wenzel A, Kostopoulos L, and Karring T."Bone healing and soft tissue contour changes following singletooth extraction: a clinical and radiographic 12-month prospective study," International Journal of Periodontics and Restorative Dentistry. 2003; 23: 313-23

4. Brägger U. Radiographic parameters: biological significance and clinical use. Periodontol 2000. 2005; 39: 73-90.

5. Mol A. Imaging methods in periodontology. Periodontol 2000. 2004; 34: 34-48.

6. Scarfe WC, Farman AG, Sukovic P. Clinical applications of conebeam computed tomography in dental practice. $\mathrm{J}$ Can Dent Assoc 2006; 72: 75-80.

7. Scarfe WC, Farman AG. What is Cone- beam CT and how does it work? Dent Clin North Am. 2008; 52: 707-30.

8. Schultze R, Heil U, Grob D, et al. Artifacts in CBCT: a review. DentomaxillofacRadiol 2001; 40: 265-73.

9. Pauwels R, Araki K, Siewerdsen JH, Thongvigitmanee SS. Technical aspects of dental CBCT: state of the art. Dentomaxillofac Radiol. 2015;44:20140224

10. Scarfe WC, Li Z, Aboelmaaty W, Scott SA, Farman AG. Maxillofacial cone beam computed tomography: essence, elements and steps to interpretation. Aust Dent J. 2012; 57:46-60.

11. Misch KA, Yi ES, Sarment DP. Accuracy of cone beam computed tomography for periodontal defect measurements. J Periodontol. 2006; 77:61-6.

12. Mengel R, Candir M, Shiratori K, Flores-de-Jacoby L. Digital volume tomography in the diagnosis of periodontal defects: an in vitro study on native pig and human mandibles. J Periodontol. 2005; 76: 65-73.

13. Vandenberghe B, Jacobs R, Yang J. Detection of periodontal bone loss using digital intraoral and cone beam computed tomography images: an in vitro assessment of bony and/or infrabony defects. Dentomaxillofac Radiol. 2008; 37:252-60.

14. Vandenberghe B, Jacobs R, Yang J. Diagnostic validity (or acuity) of 2D CCD versus 3D CBCT-images for assessing periodontal breakdown. Oral Surg Oral Med Oral Pathol Oral Radiol Endod. 2007; 104:395-01
15. El Zoheiry HS, Abou-Khalaf A, Farid MM. Assessment of periodontal defects using cone beam computed tomography. An in-vitro study. Egyptian Journal of Oral and Maxillofacial Surgery 2011, 2:27-33.

16. Caldas Mde P1, Ramos-Perez FM, de Almeida SM, HaiterNeto F. Comparative evaluation among different materials to replace soft tissue in oral radiology studies. J Appl Oral Sci. 2010; 18: 64-7.

17. De Vos W, Casselman J, Swennen GR. Cone-beam computerized tomography (CBCT) imaging of the oral and maxillofacial region: a systematic review of the literature. Int J Oral Maxillofac Surg.2009; 38: 9-25.

18. du Bois AH, Kardachi B, Bartold PM. Is there a role for the use of volumetric cone beam computed tomography in periodontics? Aust Dent J. 2012; 57: 3-8

19. Raichur PS, Setty SB, Thakur SL, Naikmasur VG. Comparison of radiovisiography and digital volume tomography to direct surgical measurements in the detection of infrabony defects. J Clin Exp Dent. 2012; 4:e43-7.

20. Takeshita WM, Vessoni Iwaki LC, Da Silva MC, Tonin RH. Evaluation of diagnostic accuracy of conventional and digital periapical radiography, panoramic radiography, and cone-beam computed tomography in the assessment of alveolar bone loss. Contemp Clin Dent 2014; 5:318-23.

21. Grimard BA, Hoidal MJ, Mills MP, Mellonig JT, Nummikoski PV, Mealey BL. Comparison of clinical, periapical radiograph, and cone-beam volume tomography measurement techniques for assessing bone level changes following regenerative periodontal therapy. J Periodontol. 2009; 80:48-55.

22. Abdel-Rahim D.F, Saleh H.A, El-Ashiry S.G. Diagnostic validity of cone beam computed tomography versus digital intraoral radiography for assessment of alveolar bone loss. Egyptian Dental Journal. 2014; 60: 89-99.

23. Al-Rawi B, Hassan B, Vandenberge B, Jacobs R: Accuracy assessment of three-dimensional surface reconstructions of teeth from cone beam computed tomography scans. J Oral Rehabil. 2010; 37: 52-8,

24. Kamburoğlu K, Murat S, Kılı̨̧ C, Yüksel S, Avsever H, Farman A and Scarfe W C. Accuracy of CBCT images in the assessment of buccal marginal alveolar peri-implant defects: effect of field of view. Dentomaxillofacial Radiology (2014); 43, 20130332.

25. Cook VC, Timock AM, Crowe JJ, Wang M, Covell DA Jr. Accuracy of alveolar bone measurements from cone beam computed tomography acquired using varying settings. Orthod Craniofac Res. 2015;18: 27-36 